\title{
Spontaneous recovery after training with multiple outcomes
}

\author{
ROBERT A. RESCORLA \\ University of Pennsylvania, Philadelphia, Pennsylvania
}

\begin{abstract}
Four experiments explored the increase in an instrumental response ( $R$ ) with time after it had been subjected either to extinction or to training with a second outcome $(O)$. Experiment 1 found less performance of an extinguished response immediately after extinction than after a 7-day delay (spontaneous recovery). Experiments $2-4$ found a similar difference when training with a second outcome replaced extinction, despite the failure of that replacement to itself undermine performance. Similar results did not occur when the second outcome was identical to that used in initial training. These results suggest that training with a novel outcome generates a decremental process that is not directly observable but that dissipates with time.
\end{abstract}

Instrumental training, in which a response $(\mathrm{R})$ produces an appetitive outcome $(\mathrm{O})$, typically results in the formation of an $\mathrm{R}-\mathrm{O}$ association. Recent results suggest that this association, once formed, is relatively impervious to attempts at its removal. For instance, simple nonreinforced occurrence of the response, arranging for the response to be independent of the outcome, or the reinforcement of the response with a second outcome have proven ineffective in undermining the $\mathrm{R}-\mathrm{O}$ association (e.g., Rescorla, 1991, 1992). Although such procedures as extinction or response-independent outcome delivery result in reductions in response probability, focused assessments have found no reduction in the strength of the $\mathrm{R}-\mathrm{O}$ association.

Those data suggest that performance reductions are the result of some decremental process that is independent of the particular outcome with which the response is associated. For instance, it may be that nonreinforced responding results in the anticipation of outcomes that fail to occur, resulting in frustration (e.g., Amsel, 1958). That frustration $(\mathrm{F})$ might in turn become associated with the response, yielding an $\mathrm{R}-\mathrm{F}$ association. Such an $\mathrm{R}-\mathrm{F}$ association might be superimposed on a relatively intact $\mathrm{R}-\mathrm{O}$ association in such a way as to undermine performance.

The present experiments focused on a potential disrupter of an initially trained $\mathrm{R}-\mathrm{O} 1$ association that does not result in performance decrement: reward of $\mathrm{R}$ with a different outcome, O2. Several experiments have suggested that such training with $\mathrm{O} 2$ leaves the $\mathrm{R}-\mathrm{O} 1$ association essentially unchanged (Rescorla, 1991, 1993). These experiments have measured the $\mathrm{R}-\mathrm{Ol}$ association in two ways: by examining the depression of $\mathrm{R}$ when $\mathrm{O} 1$

This research was supported by National Science Foundation Grants BNS-88-03514 and IBN94-04676. Correspondence concerning this article should be addressed to R. A. Rescorla, Department of Psychology, University of Pennsylvania, 3815 Walnut St., Philadelphia, PA 19104. is devalued through pairing with the toxin $\mathrm{LiCl}$, and by examining the augmentation of $R$ produced by a stimulus (S) that has elsewhere signaled O1. Both of these are well-documented procedures for detecting the presence of an R-O1 association (Colwill \& Rescorla, 1985, 1988). However, both have found the R-Ol association to be of the same strength whether or not $R$ has subsequently been trained with O2 in place of O1 (Rescorla, 1991, 1993). Indeed, some experiments suggest that successively training $\mathrm{R}$ first with $\mathrm{O} 1$ and then with $\mathrm{O} 2$ results in $\mathrm{R}$ having essentially equivalent associations with $\mathrm{O} 1$ and $\mathrm{O} 2$ (Rescorla, 1993).

However, another observation makes the concurrent presence of both $\mathrm{R}-\mathrm{O} 1$ and $\mathrm{R}-\mathrm{O} 2$ somewhat puzzling. When equivalent $\mathrm{O} 1$ and $\mathrm{O} 2$ outcomes are used, $\mathrm{R}$ 's rate of occurrence is almost the same after simple $\mathrm{O} 1$ training as it is after training with both $\mathrm{O} 1$ and $\mathrm{O} 2$ (e.g., Rescorla, 1991). Why does not the concurrent presence of $\mathrm{R}-\mathrm{O} 1$ and $\mathrm{R}-\mathrm{O} 2$ yield a greater response rate than does R-O1 alone? One possibility is that the decremental process that accounts for rate reduction in extinction is also active when a response is followed by a second outcome. For instance, it could be that during reinforcement by $\mathrm{O} 2$, the $\mathrm{R}$ becomes associated both with $\mathrm{O} 2$ and with $F$. This might occur if $F$ developed to the degree that the value of the obtained outcome was less than that anticipated on the basis of the combined $\mathrm{R}-\mathrm{O} 1$ and $\mathrm{R}-\mathrm{O} 2$ associations. Although the response generates the anticipation of both $\mathrm{O} 1$ and $\mathrm{O} 2$, only $\mathrm{O} 2$ is actually delivered.

If an account such as this has merit, then one should be able to identify some common phenomena resulting from extinction and from reinforcement by a second outcome. The present experiments focused on one possible phenomenon: spontaneous recovery.

It is well documented that a response that has been depressed by extinction will, with the passage of time, recover some of its likelihood of occurrence. Such spontaneous recovery has conventionally been interpreted in terms of the dissipation of some decremental process that 
was superimposed on the original learning. In the present context, spontaneous recovery might represent the weakening of the $\mathrm{R}-\mathrm{F}$ association so as to allow the continued $\mathrm{R}-\mathrm{O} 1$ association to be exhibited in performance again. If the same $R-F$ association develops during the training of $\mathrm{R}$ with $\mathrm{O} 2$ after initial $\mathrm{R}-\mathrm{O} 1$ training, then one would anticipate weakening of $\mathrm{R}-\mathrm{F}$ in that instance as well. Consequently, although training with $\mathrm{O} 2$ would not augment responding immediately, it might do so after a period of time. With such a delay, the concurrently present $\mathrm{R}-\mathrm{O} 1$ and $\mathrm{R}-\mathrm{O} 2$ associations would have less depressive $\mathrm{R}-\mathrm{F}$ association opposing them and hence could together generate more responding than would a simple $\mathrm{R}-\mathrm{O} 1$ association.

This possibility was explored in four experiments. Experiment 1 documented the occurrence of spontaneous recovery after simple extinction. It used a novel withinsubject design that is especially convenient for subsequent analysis. Experiment 2 repeated Experiment 1, but with $\mathrm{R}-\mathrm{O} 2$ training instead of extinction. Experiments 3 and 4 were more elaborate within-subject experiments that addressed various alternative interpretations.

\section{EXPERIMENT 1}

Experiment 1 was designed to assess spontaneous recovery after extinction of an instrumental response, using the present procedures. The conventional spontaneous recovery study involves training a response, extinguishing it, waiting some period of time, and then testing the response. The observation of interest is that responding is greater after the waiting period than it was at the end of extinction. Unfortunately, that procedure involves a comparison of responding under circumstances that differ in a number of ways other than in the time since extinction. They also differ in the time since original training, the overall age of the animal at the time of the test, and the number of previous extinction exposures (because the last observation taken at the end of extinction necessarily involves an additional extinction trial). Although there is likely to be no way to remove all such confoundings, the present experiment used an alternative within-subject procedure, illustrated in Figure 1 , that offers some advantages. In this design, each rat is trained to make two instrumental responses, R1 and R2. Extinction is then given either immediately after training (for R1) or after a delay (for R2). Both responses are tested for recovery immediately after the ex-

\begin{tabular}{l|l|l|l|l} 
Train & Ext1 & Rest & Ext2 & Test \\
\hline R1-01 & R1- & & & R1 \\
R2-01 & & & R2- & R2
\end{tabular}

Figure 1. Design of Experiment 1. After initial training of two responses, one was extinguished immediately and one was extinguished after a delay. Both responses were tested immediately after the second extinction. tinction of R2. This procedure of testing both responses at the same time allows one to compare recovery for responses that differ in their time since extinction while sharing the same time since training. It also controls for any general changes in the animal or overall responding with the passage of time. Furthermore, it removes any difference in overall extinction exposure for responses tested immediately and after a delay.

\section{Method}

\section{Subjects and Apparatus}

The subjects were 16 male Sprague-Dawley rats about 90 days old. They were housed in individual cages and maintained on a food-deprivation regime that kept them at $80 \%$ of their ad-lib body weights. They had free access to water in the home cages.

The apparatus consisted of eight operant chambers measuring $22.9 \times 20.3 \times 20.3 \mathrm{~cm}$, identical to those used in previous reports (e.g., Colwill \& Rescorla, 1985). The two endwalls of each chamber were aluminum; the sidewalls and ceiling were clear Plexiglas. Each chamber had a recessed food magazine in the center of one endwall. Two small metal cups measuring $1.25 \mathrm{~cm}$ in diameter and $1.5 \mathrm{~cm}$ deep were sunk side by side in the floor of each food magazine. To the left of the magazine was a lever, and to the right was a chain suspended from a microswitch mounted on the lid of the chamber. Located directly above the food magazine was a $2-\mathrm{cm}$ opening, behind which was an aluminum plate that activated an at tached microswitch when displaced by a nosepoke. Inserted under the grid floor, just to the right of the magazine aperture, was a flat metal rod, one end of which was bent back to form a handle. An upward pull on this handle operated a microswitch. Access to these manipulanda could be blocked by covering the lever with a metal shield, retracting the chain through a hole in the ceiling, covering the nosepoke opening with a jeweled lens, and removing the handlepull from under the grid floor. The floor of the chamber was composed of $0.48-\mathrm{cm}$ stainless steel rods, spaced $1.9 \mathrm{~cm}$ apart Each chamber was enclosed in a sound- and light-resistant shell The outside ceiling of the shell supported two solenoid-operated gravity feed valves that were connected via plastic tubing to the cups in the food magazine. One system permitted the presentation of $0.3 \mathrm{ml}$ of an $8 \%$ sucrose solution; the other permitted the presentation of $0.3 \mathrm{ml}$ of a $15 \%$ Polycose solution. Also attached to the food magazine was a dispenser containing 45-mg pellets ( $P . J$ Noyes Co., Formula A).

Experimental events were controlled and recorded automatically by relays and microprocessors located in an adjoining room.

\section{Procedure}

Initial training. On the 1 st day, the animals received a $20-\mathrm{min}$ magazine training session containing 20 pellet deliveries. Over the next 2 days, all animals were trained to leverpress and nosepoke with a pellet reinforcer. Each training session allowed responding to earn 25 deliveries of the pellets on a continuous reinforcement schedule. Throughout this initial training, individual shaping was used if necessary for a particular response. On each of the next 5 days, the animals received two 20 -min training sessions spaced about $30 \mathrm{~min}$ apart. Each session contained one manipulandum on which responding was reinforced by pellets on a variable-interval (VI) schedule with a mean of $1 \mathrm{~min}$.

Extinction. On each of the next 2 days, all animals received extinction with one of the responses. Each session was $20 \mathrm{~min}$ in duration, and no pellets were delivered. For half of the animals, the extinguished response was leverpressing; for the other half, it was nosepoking. On each of the next 5 days, the animals were maintained on a deprivation schedule but did not leave their home cages. On the following 2 days, each animal received a 20 -min extinction session with its alternative response. 
Recovery test. Immediately following the second session of extinction of the second response, each animal was subjected to two short test sessions, one with each response. At the end of the extinction, and again at the end of the first test, the animals were removed from the chambers, the appropriate manipulanda were installed, and the animals were returned to the chamber. The process of changing manipulanda took approximately $5 \mathrm{~min}$. For half of the animals, the first-tested response was leverpress; for the other half, it was the nosepoke. For half of each of those subgroups, the firsttested response was that which had just been extinguished; for the other half, it was that which had been extinguished 7 days earlier. Each test session was 8 min long.

\section{Results and Discussion}

Initial training proceeded without incident. On the final day of training, the mean responses per minute were 17.1, with no reliable differences as a function of response identity. Extinction proceeded similarly for both responses, with the mean responses per minute being 6.1 and 5.3 for $R 1$ and $R 2$, respectively. During the final 5-min period of extinction, the mean responses per minute were 2.0 and 0.9 for R1 and R2, respectively. Although somewhat more responding occurred with the response extinguished first, there were no reliable differences.

Figure 2 displays responding over the course of the test, separated for the response that was first extinguished ( 7 days earlier) or second extinguished (minutes earlier). Because the order of testing had no reliable effect, performance has been combined across that variable. It is clear that the two responses differed substantially, with considerably more performance on the response that had been extinguished less recently. Over the first half of the 8-min session, total responding was reliably greater on the response with the opportunity for recovery [Wilcoxon $T(15)=3.5, p<.01]$.

These results document the occurrence of spontaneous recovery with this experimental procedure. When two responses were tested at the same distance from training but different distances from extinction, they showed quite different levels of performance. There was greater recovery from the depressive effects of extinction

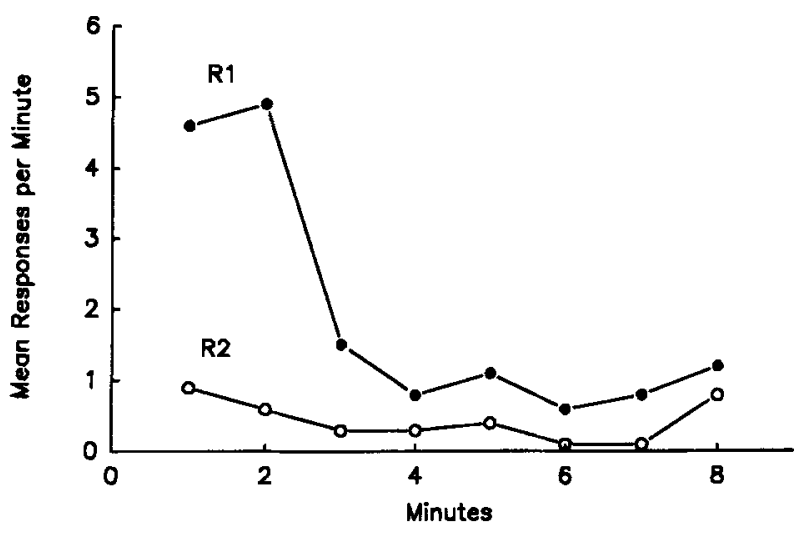

Figure 2. Performance during the test session of Experiment 1. One response (R2) had been extinguished immediately prior to the test; the other response (R1) received its extinction 7 days earlier. with the greater passage of time. Moreover, the specificity of the recovery to a particular response suggests that what is recovering is not some general responseindependent process, such as motivation or reduction of fatigue. The present recovery is specific to the particular response or to the particular response-outcome relation, not general to responses trained with that outcome.

\section{EXPERIMENT 2}

Experiment 2 was modeled after Experiment 1, except that nonreinforcement was replaced with $\mathrm{O} 2$ in the second "extinction" phase. Each animal was trained with two responses ( $R 1$ and $R 2$ ) earning liquid sucrose. Both responses were then trained with pellet reward, with $R 1$ receiving that training immediately and $\mathrm{R} 2$ receiving it after a delay. Both responses were then tested in extinction. If training with pellets has resulted in a depressive process that dissipates with time, performance during the test should be higher for the response that had its pellet training earlier, relative to that receiving its pellet training more recently.

Sucrose and pellets were deliberately assigned the roles of $\mathrm{O} 1$ and $\mathrm{O} 2$ in this experiment. Prior experiments had suggested that sucrose often supports an overall lower response rate than do pellets. It seemed important, for the initial investigation, to be sure that the reinforcer delivered in the role of $\mathrm{O} 2$ would not reduce responding, relative to that supported by the initial 01 . Such a reduction could mean that $\mathrm{O} 2$ had a lower reinforcing value than $\mathrm{O} 1$ and that its use effectively submits the responses to a form of extinction through reinforcer value reduction. In that case, it would hardly be surprising if spontaneous recovery occurred to restore responding toward the level supported by O1. Consequently, the issue in Experiment 2 was whether responding will be higher when a longer time has intervened between $\mathrm{R}-\mathrm{O} 2$ training and test, under circumstances in which $\mathrm{R}-\mathrm{O} 2$ training itself does not lower performance, relative to the prior $\mathrm{R}-\mathrm{O} 1$ training.

\section{Method}

\section{Subjects and Apparatus}

The subjects were 16 naive rats of the same stock and were maintained in the same manner as those in Experiment 1. The apparatus was the same as that of Experiment 1.

\section{Procedure}

Initial training. All animals received two magazine sessions like that given in Experiment 1. Each session contained 20 deliveries of an outcome given over $20 \mathrm{~min}$. The first session contained pellets, and the second contained deliveries of $0.3 \mathrm{ml}$ of $8 \%$ sucrose solution. Over the next 2 days, all animals were trained to leverpress and nosepoke for sucrose, on a continuous reinforcement schedule. They then received five 20 -min VI training sessions, identical to those given in Experiment 1 except that sucrose was the reinforcer.

Training with $\mathrm{O} 2$. On each of the next 2 days, all animals received a 20-min training session with one response. For half of the animals, that response was the leverpress; for the other half, it was the nosepoke. During these sessions, all animals earned pellets on a VI 1-min schedule. On each of the next 5 days, the animals remained on a de- 
privation schedule in their home cages. On the next 2 days, each animal received a VI training session with its alternative response.

Testing. Immediately after the second training session with the second response, all animals were tested. The testing procedure was identical to that used in Experiment 1: two 8-min extinction sessions with the order of the responses counterbalanced.

\section{Result and Discussion}

Initial training was uneventful. On the final day of training with sucrose, the mean responses per minute were 8.4 , with no difference between responses. Over the course of training with pellets, the mean responses per minute were 10.2 and 11.9 for the responses trained immediately and with delay, respectively; the response rates were 13.3 and 15.3 for those two responses in the final $5 \mathrm{~min}$ of training with pellets. Neither of these differences was reliable; however, by the end of training, both $\mathrm{R} 1$ and $\mathrm{R} 2$ showed increased responding, relative to their rates when reinforced with sucrose $[T \mathrm{~s}(16)=16.6$ and $6, p s<.02]$.

Figure 3 shows the course of responding during the 8 -min extinction test session. Naturally, responding declined over the course of the extinction test. But the performance was higher on the response trained with pellets farther in the past (R1). As one might expect, this superiority was evident early in the session and then was reduced with extinction testing. Over the first half of the session, the response trained earlier showed reliably greater performance than did the response trained more proximal to the test $[T(16)=10.5, p<.01]$.

These results suggest that there is improvement in responding with the passage of time since reinforcement with a second outcome. This observation is consistent with the notion that training with a second outcome engenders some unobserved depressive process that dissipates with the passage of time.

The measurement of two responses at the same time within the same animal seems to rule out some alternative interpretations. For instance, it seems unlikely that the difference between performance on the responses

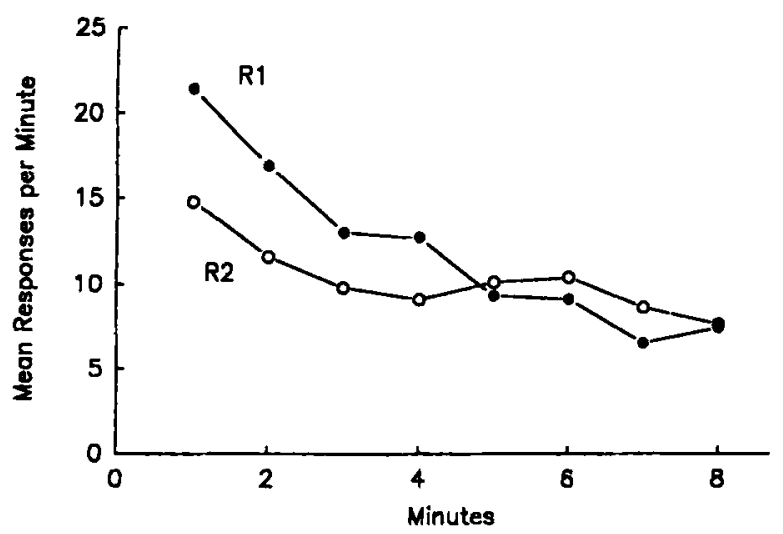

Figure 3. Performance during the test session of Experiment 2. One response (R2) had been followed by a new outcome immediately prior to the test; the other response (R1) had that training 7 days earlier. trained with $\mathrm{O} 2$ proximal and distal to the test is attributable to differences in motivation. Both responses had been reinforced with the same outcomes. Moreover, differential responding could not be attributed to general changes in the animal as a function of time.

However, other interpretations, such as differential fatigue or reactive inhibition, Ir (Hull, 1943) for the two responses, remain viable. According to those views, an animal might be generally less likely to make a response that it has recently made, because of some response-specific inhibition. This account focuses on the response, rather than on the response-outcome relation. For this reason, it anticipates the same result whatever outcome was used in the additional training of the response. By contrast, according to the view being explored here, it is critical to use an $\mathrm{O} 2$ in the additional training that differs from the $\mathrm{O} 1$ of original training. The different $\mathrm{O} 2$ results in additional associative strength, which in turn summates with the original $\mathrm{R}-\mathrm{O} 1$ association and leads to an anticipation that is inappropriately large for the $\mathrm{O} 2$ reinforcement conditions in effect. Consequently, Experiments 3 and 4 compared the effects of immediate or delayed test when $\mathrm{O} 2$ was either different from or the same as $\mathrm{O} 1$.

Experiments 3 and 4 also corrected an obvious shortcoming of Experiment 2: the identification of $\mathrm{O} 1$ with a particular reinforcer, sucrose, and $\mathrm{O} 2$ with a particular different reinforcer, pellets. It seems important in pursuing these observations to counterbalance fully the outcome identifications. Experiments 3 and 4 did that.

\section{EXPERIMENT 3}

Experiment 3 was an elaboration of Experiment 2. Two groups of rats received training of $\mathrm{R} 1$ and $\mathrm{R} 2$, but with different outcomes, as illustrated in Figure 4. Both groups then received additional training of one response, followed by a delay, followed by training of the other response. Immediately after the additional training of the second response, both responses were tested in extinction. For Group D (different), each response received its additional training with a different outcome; for Group S (same), each response received its additional training with the same outcome. However, the design ensured that all animals had equivalent experience with each of the individual responses and outcomes. The treatment of Group D constituted a replication of Experiment 2 with counterbalanced outcome identities. The treatment of Group $S$ provided a comparison when the responses were trained only with one outcome.

\section{Method}

\section{Subjects and Apparatus}

The subjects were 16 naive rats of the same stock and were maintained in the same manner as those in Experiment 1. The apparatus was the same as that of Experiment 1.

\section{Procedure}

Initial training. All animals received two magazine sessions like those given in Experiment 2 . They then received training to leverpress and nosepoke, with the sucrose and pellet outcomes counter- 


\begin{tabular}{c|c|c|c|c|c} 
& Train & Troin 2nd 0 & Rest & Train 2nd O & Test \\
\hline Group D & $\mathbf{R} 1-01$ & $\mathbf{R} 1-02$ & & & $\mathbf{R 1}$ \\
& $\mathbf{R 2 - 0 2}$ & & & $\mathbf{R 2 - 0 1}$ & $\mathbf{R 2}$ \\
\hline \multirow{2}{*}{ Group S } & $\mathbf{R} 1-01$ & $\mathbf{R} 1-01$ & & & $\mathbf{R}$ \\
& $\mathbf{R 2 - 0 2}$ & & & $\mathbf{R 2 - 0 2}$ & $\mathbf{R 2}$
\end{tabular}

Figure 4. Design of Experiment 3. All animals received training of two responses (R1 and R2) with different outcomes (O1 and $\mathrm{O2}$ ). Each response then received additional training immediately or after a delay. For Group D, different outcomes were used during this additional training; for Group S, the same outcomes continued. Both groups were tested immediately after the training of the second response.

balanced across animals and across responses. On each of the next 5 days, each animal received two 20 -min VI sessions, one with leverpress and one with nosepoke. The earned outcomes were the same as those used in initial training.

Training with second outcome. On each of the next 5 days, each animal received one 20 -min VI session, with either leverpress or nosepoke. For the animals in Group D, the earned outcome was the alternative to that earned in initial training. For the animals in Group S, it was the same outcome. On each of the next 5 days, all animals remained on deprivation but were not placed in the experimental chambers. On the next 5 days, each animal received a VI training session with the alternative response. For Group D, the outcome again was different from that used in initial training; for Group S, it was the same.

Test. Immediately after the last session of this additional training, all animals received two 8-min test sessions, one with nosepoke and one with leverpress. The order of responses was counterbalanced by manipulandum identity and prior treatment.

\section{Results and Discussion}

Initial response training proceeded smoothly. On the final day of initial training, the mean responses per minute were 19.5 and 19 for the responses to be given immediate and delayed training with the same outcome, respectively. The comparable response rates were 20.5 and 19.9 for the responses to be given immediate and delayed training with the different outcome. None of these differences were reliable. However, responding reinforced by pellets ( 27.8 per minute) again proved greater than that reinforced by sucrose ( 11.7 per minute) $[T(16)=$ $16, p<.01]$.

The course of training with the second outcome was similar when given immediately or with a delay. For that phase as a whole, the mean responses per minute were 20.2 and 19.4 for the responses trained immediately and with a delay, respectively. On the final day of immediate and delayed training with the additional reinforcer, the mean responses per minute were 21.8 and 17.7 , respectively. Although in both cases pellets supported higher response rates, in neither case was the difference reliable. The results of most interest, from the test sessions, are shown in Figures 5 and 6.

Figure 5 shows responding over the course of testing. It separates responses tested immediately after second outcome training (R2) from those tested after a delay
(R1) in Groups S and D. Examination of the open and filled circles makes it clear that in Group D there was greater performance on the response that had more opportunity for recovery (R1). This difference was especially evident at the beginning of the session and declined with extinction testing. By contrast, examination of the open and filled squares indicates that Group S showed greater performance on the response that was more recently trained (R2).

The difference between the groups is further highlighted in Figure 6, which displays responding over the first half of the session, expressed as a percentage of the responding at the end of initial $\mathrm{R}-\mathrm{O} 1$ training, prior to the differential treatments. This measure reduces the variability attributable to individual differences in performance with different animals and outcomes. The results of Group D, displayed at the left, replicate those of Experiment 2. There was greater performance on the response that received training with a second outcome more distal from the test $[T(8)=3, p<.05]$. The results

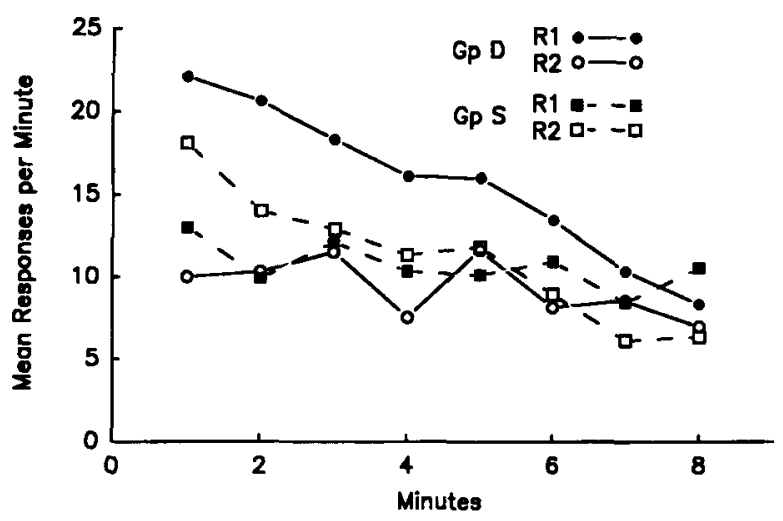

Figure 5. Performance during the test sessions of Experiment 3. Response and group identifications are as in Figure 4.

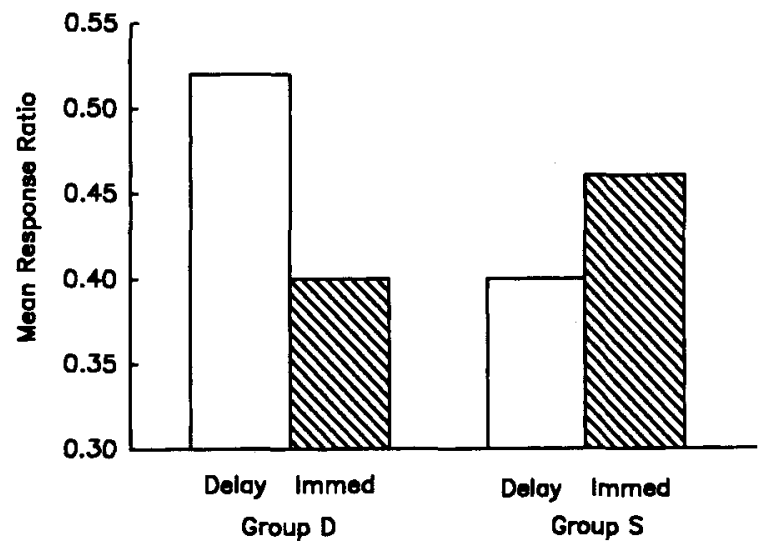

Figure 6. Performance during the first half of the test sessions in Experiment 3, plotted as a ratio of responding at the end of initial training. Responding is shown separately for behaviors tested immediately or with a delay after their second training. 
for Group S, displayed at the right, show quite a different pattern. For these animals, performance was greater for the response tested immediately after training, although the difference was not reliable. The greater superiority of delayed over immediate testing in Group D relative to Group $\mathrm{S}$ was also reliable $[U(8,8)=13, p<.05]$.

These results suggest that the recovery shown following training of a response with two outcomes depends on those outcomes having different identities. This implies that it is not attributable to some property of the response execution per se, such as fatigue or Ir, but rather depends on multiple $\mathrm{R}-\mathrm{O}$ associations.

\section{EXPERIMENT 4}

Experiment 3 compared two groups that differed in whether their responses were successively trained with the same or different outcomes. Although that experiment matched the groups on exposure to the individual responses and outcomes, it did not match them on their exposure to a shift in reinforcement conditions. Animals in Group $\mathrm{D}$ experienced a change in their $\mathrm{R}-\mathrm{O}$ relations, whereas animals in Group S did not. Consequently, Experiment 4 attempted to repeat the observations made in Experiment 3, but using a fully within-subject design that exposed all animals to a reinforcement shift.

For this purpose, each animal was trained with four responses: leverpress, nosepoke, handlepull, and chainpull. For each animal, two responses then received immediate additional training and two received delayed additional training. For each of these pairs of responses, one received its additional training with the same outcome and one with a different outcome. This permitted the comparison of all four of the treatments of Experiment 3 in the same animal, thus ensuring equivalence of overall treatment.

\section{Method}

Subjects and Apparatus

The subjects were 16 naive rats of the same stock and were maintained in the same manner as those in Experiment 1 . The apparatus was the same as that of Experiment 1.
}

\section{Procedure}

Initial training. All animals received two magazine sessions like those given in Experiment 2. They then received training to leverpress, nosepoke, handlepull, and chainpull, with the sucrose and pellet outcomes counterbalanced across manipulanda. On each of the next 10 days, each animal received two 20 -min VI sessions. On 5 of those days, they received one session with leverpress and one with chainpull; on the other 5 days, they received one session with nosepoke and one with handlepull. The earned outcomes were the same as those used in initial training.

Training of R1 and R2 with second outcome. On each of the next 2 days, each animal received one 20 -min VI session, with one of the manipulanda and with the outcome either the same as or different from that used in initial training. The selection of manipulanda and outcome was counterbalanced. On each of the next 5 days, all animals remained on deprivation but were not placed in the experimental chambers. On the next 2 days, each animal received a VI training session with a second response. Again, the outcome was either the same as or different from that used in initial training.

Test. Immediately after the 2 nd day of this additional training, all animals received two 8-min test sessions, one with each additionally trained response. The order of responses was counterbalanced by manipulandum identity and prior treatment.

Training of R3 and R4 with second outcome. On the next 2 days, the entire cycle of training with $\mathrm{O} 2$ was repeated for the other two responses.

The result of these treatments is that each animal has four responses, two tested immediately after the second training and two after a delay; one of each of these response pairs was trained with one outcome, and one of each was trained with two different outcomes.

\section{Results and Discussion}

Initial training was smooth. There were no systematic differences between the pair of responses given training with the second outcome and testing first and the pair given that experience second. On the final day of initial training, the mean responses per minute were 16.0 and 13.6 for the responses to be given second-outcome training with the same outcome immediately and with a delay, respectively. The comparable numbers were 13.3 and 12.9 for the responses to be given second-outcome training with the different outcome. Over the course of training with the second outcome, the mean responses per minute were 12.3 and 12.5 for the responses trained immediately and with a delay; the comparable numbers for the last day of such training were 10.8 and 13.3. In neither case was the difference reliable. Consequently, Figure 7 combines the results of the two tests in the manner of Figure 6.

These results show that when a response is trained with two different outcomes, there is greater performance if testing occurs after a delay rather than immediately $[T(16)=24.5, p<.05]$. On the other hand, there is no evidence for a similar superiority of delayed testing when training is with the same outcome. The difference between immediate and delayed test proved reliably greater in the case of using different outcomes $[T(16)=$ $26, p<.05$ ].

\section{GENERAL DISCUSSION}

These experiments provide evidence for a growth in the performance of a response as time passes after its training with a second outcome. This observation is similar to that of spontaneous recovery of responding with the passage of time after extinction. It is consistent with the view that training with a second outcome not only strengthens the association of the outcome with the response but also produces a depressive process that dissipates with time.

These experiments failed to show a similar pattern when additional training was done with the same outcome that originally reinforced the response. Indeed, under those circumstances, there is the suggestion that responding may be greater when testing takes place immediately after the additional training rather than after a 


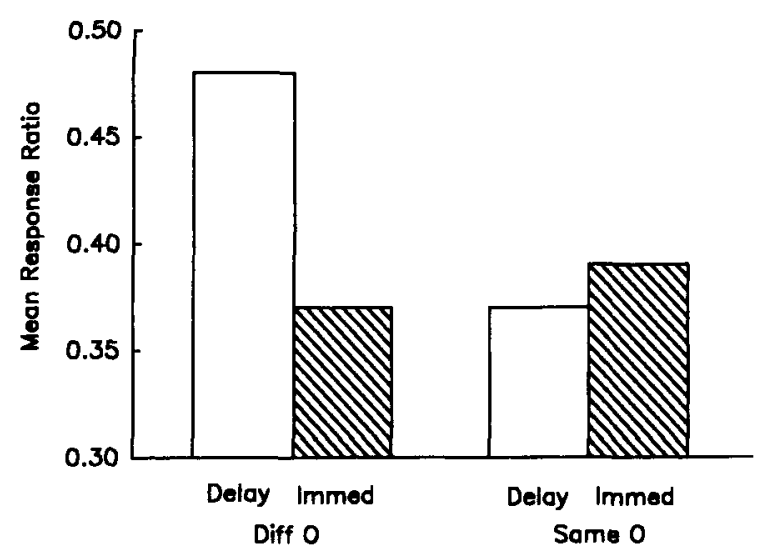

Figure 7. Performance during the first half of the test sessions of Experiment 4, plotted as a ratio of responding at the end of initial training. Responding is shown for behaviors tested immediately or with a delay after their second training, separated according to whether that second training involved the same or a different outcome.

delay. It may be that when only one reinforcer is used, recent rewarded execution of a response primes it for subsequent occurrence. If so, then the opposite finding when different reinforcers are used must be evaluated against this baseline.

The results of these experiments suggest a commonality between the consequences of extinction and reinforcement with an alternative reinforcer. However, they do not uniquely identify the nature of the process that they share. On the basis of earlier work, the present discussion has favored some outcome-independent depressive process, such as the formation of an association between the response and frustration. In that view, extinction leaves the $\mathrm{R}-\mathrm{O} 1$ association intact and superimposes a depressive process, such as an $\mathrm{R}-\mathrm{F}$ association, which does not code for a specific outcome. The extension of that view to the case of two successive outcomes is that an R-F association is superimposed on $\mathrm{R}-\mathrm{O} 1$ and $\mathrm{R}-\mathrm{O} 2$ associations. Of course, this framework is yet to provide an account of why the contribution of the R-F association decreases with time.

An alternative view that emphasizes retrieval processes (e.g., Bouton, 1993) can accommodate some of the results reported here. In that view, extinction leaves the $\mathrm{R}-\mathrm{O} 1$ association intact and superimposes a depressive process, such as an $\mathrm{R}$-null association, where null represents the no event. During extinction, the retrieval of the R-null association is favored because of its recency. However, with the passage of time retrieval becomes less biased toward the R-null association and permits some retrieval of the original $\mathrm{R}-\mathrm{O} 1$ association and thus performance.

Applied to the present procedures, this view might see training of an $\mathrm{R}$ first with $\mathrm{O} 1$ and then with $\mathrm{O} 2$ as establishing both an $\mathrm{R}-\mathrm{O} 1$ and an $\mathrm{R}-\mathrm{O} 2$ association, perhaps with no interference at the stage of learning. However, during the additional training, the retrieval of $\mathrm{R}-\mathrm{O} 2$, rather than $\mathrm{R}-\mathrm{O} 1$, would be favored. If $\mathrm{O} 1$ and $\mathrm{O} 2$ are of comparable value, then it would not be surprising if the response rate were not increased by the addition of the new association with $R$. Despite the presence of both associations, only one would be retrieved and, hence, responding would be comparable to the case in which only one were trained.

What is more problematic is the greater responding when the $\mathrm{O} 2$ training is farther in the past relative to the testing. Although that greater time interval might encourage more balanced retrieval of $\mathrm{R}-\mathrm{O} 1$ and $\mathrm{R}-\mathrm{O} 2$, it is not clear why that should result in enhanced responding. If $\mathrm{O} 1$ and $\mathrm{O} 2$ are of comparable value, as occurs with the counterbalancing used in Experiments 3 and 4, then greater relative retrieval of $\mathrm{O} 1$, compared with $\mathrm{O} 2$, should not change the level of responding. If $\mathrm{Ol}$ controls a lower response rate than does $\mathrm{O} 2$, then the greater relative retrieval of $\mathrm{O} 1$ should result in lower responding. However, the results of Experiment 2 contradicted this prediction.

Within a retrieval view, one might not be surprised if greater responding emerged over the course of testing whatever the relative values of $\mathrm{O} 1$ and $\mathrm{O} 2$. It seems plausible that with more associations available for retrieval during testing, the animal might first respond on the basis of one $\mathrm{R}-\mathrm{O}$ association but then turn to another. However, the results of the present experiments suggest instead that the differences in response rates are greater at the beginning of the session. What is apparently needed is the specification of some rule that details how retrieval of multiple memories interacts and combines to produce performance.

The observation of increased responding with the passage of time following successive training with two outcomes suggests caution in interpreting prior experiments using a devaluation procedure. In some of those experiments (e.g., Rescorla, 1993), pairing with either the firstor the second-used outcome with $\mathrm{LiCl}$ had a similar effect on responding. This encouraged the conclusion that both $\mathrm{R}-\mathrm{O}$ associations were functional and of similar strength after response training. It should be noted, however, that the devaluation procedure itself requires more than a week, thus allowing for the possibility of recovery. Consequently, those experiments are also compatible with the conclusion that training with $\mathrm{O} 2$ temporarily depressed the $\mathrm{R}-\mathrm{O} 1$ association but that the $\mathrm{R}-\mathrm{O} 1$ association recovered with time.

In any case, the present results join others in suggesting that training with a second outcome does not destroy the initially learned $\mathrm{R}-\mathrm{O}$ association. Rather there is the superimposition of some new learning. Moreover, part of the process by which that new learning occurs is sufficiently shared with extinction to produce a phenomenon analogous to spontaneous recovery.

\section{REFERENCES}

AMSEL, A. (1958). The role of frustrative nonreward in noncontinuous reward situations. Psychological Bulletin, 55, 102-119.

Bouton, M. E. (1993). Context, time, and memory retrieval in the in- 
terference paradigms of Pavlovian learning. Psychological Bulletin, 114, 80-99.

Colwill, R. M., \& Rescorla, R. A. (1985). Post-conditioning devaluation of a reinforcer affects instrumental responding. Journal of Experimental Psychology: Animal Behavior Processes, 11, 120-132.

Colwill, R. M., \& Rescorla, R. A. (1988). Associations between the discriminative stimulus and the reinforcer in instrumental learning. Journal of Experimental Psychology: Animal Behavior Processes, 14, 155-164.

Hull, C. L. (1943). Principles of behavior. New York: AppletonCentury-Crofts.
Rescorla, R. A. (1991). Associations of multiple outcomes with an instrumental response. Journal of Experimental Psychology: Animal Behavior Processes, 17, 465-474.

RESCORLA, R. A. (1992). Response-independent outcome presentation can leave instrumental R-O associations intact. Animal Learning \& Behavior, 20, 104-111.

Rescorla, R. A. (1993). Inhibitory associations between $S$ and $R$ in extinction. Animal Learning \& Behavior, 21, 327-336.

(Manuscript received January 2, 1995; revision accepted for publication March 20, 1995.) 